\title{
Development of a motion-based cell-counting system for Trypanosoma parasite using a pattern recognition approach
}

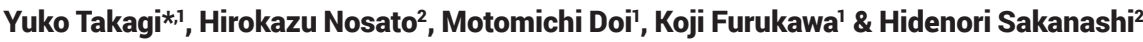

\section{ABSTRACT}

Automated cell counters that utilize still images of sample cells are widely used. However, they are not well suited to counting slender, aggregate-prone microorganisms such as Trypanosoma cruzi. Here, we developed a motion-based cell-counting system, using an image-recognition method based on a cubic higher-order local autocorrelation feature. The software successfully estimated the cell density of dispersed, aggregated, as well as fluorescent parasites by motion pattern recognition. Loss of parasites activeness due to drug treatment could also be detected as a reduction in apparent cell count, which potentially increases the sensitivity of drug screening assays. Moreover, the motion-based approach enabled estimation of the number of parasites in a co-culture with host mammalian cells, by disregarding the presence of the host cells as a static background.

\section{METHOD SUMMARY}

Cubic higher-order local auto-correlation is a method to extract features related to shape and movement of moving objects. This motion image recognition method can be applied to a cell-counting system for motile microorganisms, by learning the motion patterns observed in images of known cell densities as a training dataset to estimate the cell densities of unknown samples.

\section{KEYWORDS:}

cell count $\cdot$ Chagas disease $\cdot$ image analysis • machine learning $\bullet$ microscopy $\bullet$ pattern recognition - protozoan parasite $\cdot$ Trypanosoma cruzi 'Biomedical Research Institute, National Institute of Advanced Industrial Science and Technology, Tsukuba, Ibaraki, Japan; ${ }^{2}$ Artificial Intelligence Research Center, National Institute of Advanced Industrial Science and Technology, Tsukuba, Ibaraki, Japan; *Author for correspondence: yuko-takagi@aist.go.jp

BioTechniques 66: 179-185 (April 2019) 10.2144/ btn-2018-0163
Phenotypic analysis based on viable cell counts plays a major role in several aspects of drug development research, such as target gene validation, natural bioactive substance isolation and lead compound optimization. For a bright field cell count analysis, a variety of automated counting systems have been commercialized and implemented in a regular laboratory setting, such as TC20" (Bio-Rad, CA, USA), Countess ${ }^{\circledR}$ (Thermo Fisher Scientific, MA, USA), Luna'" (Logos Biosystem, Gyeonggi-do, South Korea), Cedex HiRes Analyzer (Roche, Basel, Switzerland), Cellometer" (Nexcelom Bioscience, MA, USA) and Vi-CELL ${ }^{\circledR}$ (Beckman Coulter, CA, USA), just to name a few. However, they are suited for round, dispersed cell types, such as mammalian cells and some unicellular organisms such as yeast, and are not well adapted to slender or aggregate-prone cells. Some instruments are equipped with declustering software to detect and split cell clumps in a captured image, but still cannot resolve densely aggregated cells and tend to misinterpret the boundaries of slender or irregular cell shapes.

Trypanosoma cruzi is the causative agent of Chagas' disease, which is prevalent in Latin America and affects 6-7 million people [1]. Since currently available drugs are accompanied with severe side effects and are ineffective in the chronic phase of the disease [2], development of new chemotherapeutics is a pressing matter. To this end, it is of a great interest to develop tools for T. cruzi research to aid in phenotypic screening.

T. cruzi has distinct life-cycle stages as it travels between the insect vector and mammalian host (reviewed in [3]). Epimastigote is the proliferation stage of $T$. cruzi in insect vectors. Since it is the most amenable stage in terms of culturing and experimental manipulation, epimastigotes are widely used to investigate the basic biology of $T$. cruzi. The most troublesome factor involved in epimastigote counting is the presence of cell aggregate, or so-called 'rosette'. Commercially and freely available image-based counting systems cannot resolve parasite aggregates, especially at a high cell density (Supplemental Figure 1), so the aggregates need to be broken apart by vigorous pipetting or shaking before the sample is applied onto a counting slide. This is troublesome in phenotypic screenings, where a typical handling format is 96- or even 384-well plates.

Another aspect of epimastigote counting involves cell motility. Since an epimastigote wiggles and swims around with flagella, cell counting by hand normally involves fixing of the cells. Commercial cell-counting systems do not necessarily require such pretreatment, but they nonetheless use a captured still image to count individual cells. This approach may lead to the loss of potentially valuable motional information in phenotypic analysis.

Here, we developed a set of software to estimate the cell counts of motile $T$. cruzi, using the motion image recognition method based on a cubic higherorder local auto-correlation (CHLAC) feature. This machine learning method allows estimation of the cell density from microscopy video images without actually counting the individual parasite. Unlike most of the commercially available counting systems, there is no need to apply samples onto specialized slides or cuvettes. The software is applicable to aggregate-rich samples and fluorescent parasites, provided that training was performed with an appropriate dataset for each situation. The 


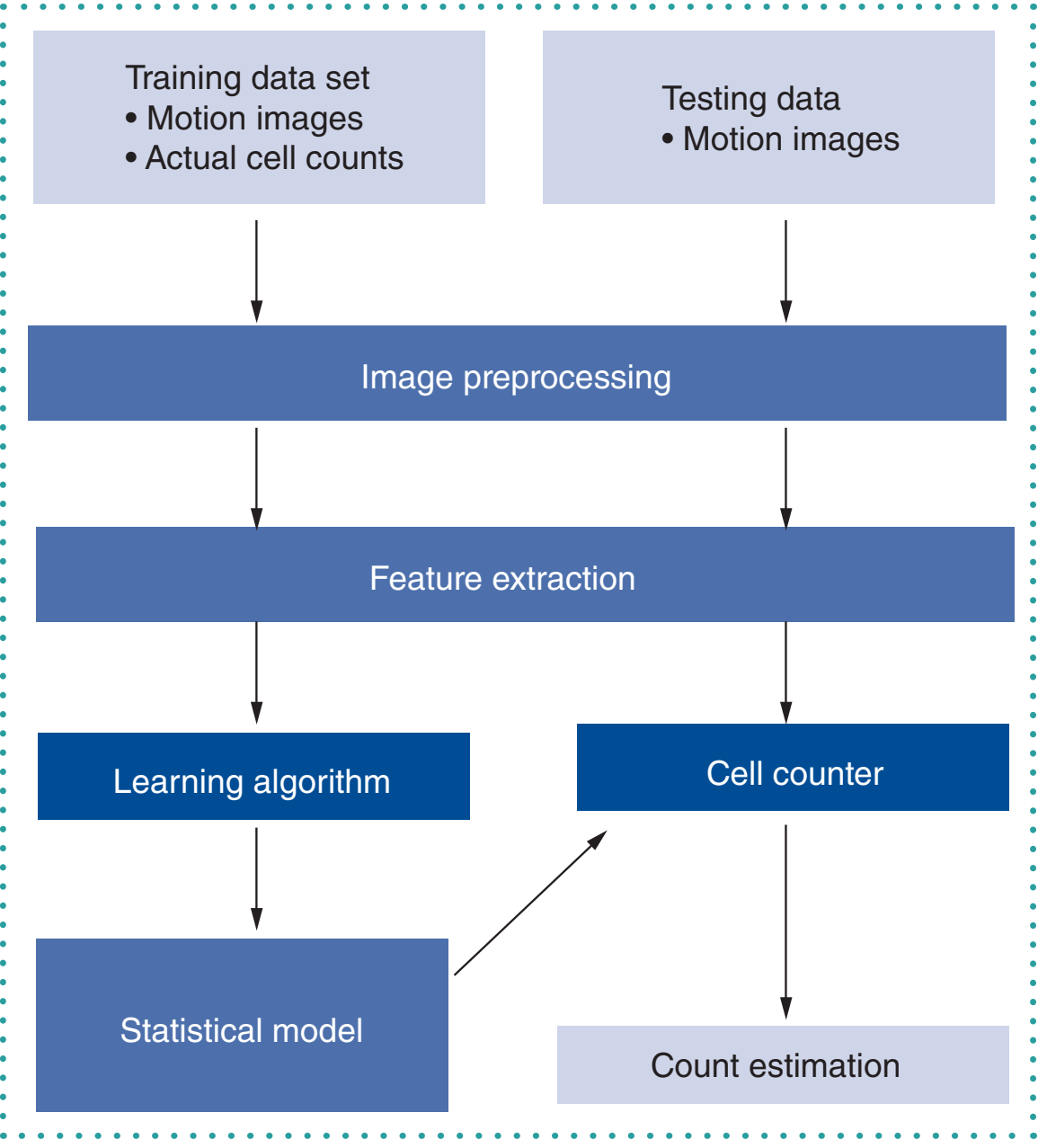

Figure 1. Overview of cell-counting system. The system consists of a pair of software; the machine learning program to develop a correlation equation of extracted features, and the parasitecounting program to estimate the cell densities of unknown samples based on the statistical model developed by the learning program.

$\checkmark$ nature of movement-based pattern-recognition enables distinction between healthy and dying parasites in drug treatment experiments. Moreover, parasites in the infectious stage can be detected and quantitated in the co-culture with host mammalian cells with no additional sample or data pretreatment.

\section{MATERIALS \& METHODS T. cruzi culture}

Epimastigotes of T. cruzi Tulahuen strain were cultured in LIT medium supplemented with $10 \%$ heat-inactivated FBS at $28^{\circ} \mathrm{C}$. mDsRedexpressing parasites were generated by transfecting pTREX-mDsRed vector into epimastigote. To construct the plasmid, theopen reading frame of $m D s R e d$ was amplified by PCR and subcloned between $X$ bal and Xhol sites of the vector derived from pTREX-n [4]. Transfection was carried out using Basic Parasite Nucleofector Kit 2
(Lonza, Basel, Switzerland) and program X-14 of Amaxa Nucleofector device (Lonza). To generate a stable cell line, the transfectants were selected in LIT medium containing $500 \mu \mathrm{g} / \mathrm{ml} \mathrm{G} 418$ for 1 month at $28^{\circ} \mathrm{C}$.

Actual cell density of the culture was determined by using a Burker-Turk hemocytometer. To capture microscopy video images for the training dataset, a dilution series was made from parasites of known cell density. A dilution series of rosette-free parasite was prepared from the culture in early-to-mid-log phase, with vigorous pipetting to break apart any cell aggregates. A dilution series of rosetterich sample was prepared from the culture in late-log to stationary phase, using gentle pipetting so as not to disturb naturally occurring parasite aggregates.

For the drug treatment experiment, nifurtimox of indicated concentration was added to the culture medium, and the parasite culture was incubated for 2 days before taking video images.

The infectious stage of T. cruzi (trypomastigote) was obtained by in vitro differentiation of epimastigotes in RPMI medium [5], followed by infection to the host 3T3-Swiss Albino fibroblast cells. The parasite-host co-culture was maintained in DMEM supplemented with $10 \% \mathrm{FBS}$ at $37^{\circ} \mathrm{C}$ under $5 \% \mathrm{CO}_{2}$. Trypomastigotes that emerged from the host cells were collected from the culture supernatant and used in the counting experiments.

\section{Image acquisition}

The parasites $(200 \mu \mathrm{l})$ were aliquoted into a 96-well clear bottom microplate, $2 \mathrm{~h}$ prior to the image capturing. Video images were taken with an all-in-one fluorescence microscope BZ-X710 (Keyence, Osaka, Japan) by using a 10X objective lens (CFI Plan Fluor DL 10x, Nikon, Tokyo, Japan). The recording setting was $1080 \times 960$ image size and the rate 15 frame per second (fps) for the bright field. Fluorescence video images were captured in $640 \times 480$ image size, and were converted to grayscale by using OpenCV library (Open Source Computer Vision Library). All the motion images were captured for $5 \mathrm{~s}$.

The close-up still images of rosette-rich epimastigote, rosette-less epimastigote and trypomastigote samples were taken with 20X objective lens (S PL FL ELWD ADM 20xC, Nikon).

\section{Motion image analysis}

Overview of machine learning and cell counting schemes is depicted in Figure 1. In the preprocessing step, frame differencing is first applied to the successive image sequences to subtract the static background and to identify moving objects in the view. Binarization with Otsu's method is then applied to emphasize the movement and to eliminate the noise below threshold [6] (Figure 2). Preprocessed images are fed into the feature extraction program to quantize the spatio-temporal correlation of the pixels in three consecutive preprocessed frames, using the motion image recognition method based on the CHLAC feature [7]. The spatial correlation contains the information on the shape and size of the objects (cells and their aggregations), and temporal correlation 
contains the information on their movements. Then, the multiple linear regression analysis is applied to derive the function (model) that integrates the values of hand-counted actual cell density and the CHLAC feature containing the information concerning direction, amount, shape and size of all movements in the frames.

The regression function is used to estimate the cell density of the testing data. The final estimated count of the cells in a video is calculated by averaging 72 estimated values predicted based on 72 CHLAC features extracted from every three consecutive frames in 74 differentiated frames made from 75 frames ( $15 \mathrm{fps} \times 5 \mathrm{~s}$ ).

The software is available from authors upon request.

\section{RESULTS \& DISCUSSION}

\section{Validation of counting program}

A training dataset for machine learning was obtained by recording video images of a dilution series of $T$. cruzi epimastigote culture. Three locations were selected in each well to account for potential variability in parasite dispersion. When the same video images used for the training dataset were analyzed as the testing data, actual cell count and machine-estimated cell count showed excellent correlation $\left(R^{2}=0.998\right)$ (Figure 3A). 'Unknown' testing samples were prepared using different dilution series from the training dataset. When those unknown samples were analyzed, machine-estimated cell count was in good agreement with the actual cell count $\left(R^{2}=0.995\right)$ (Figure $\left.3 \mathrm{~B}\right)$. This result indicates that the cell-counting method by motion image recognition based on the CHLAC feature yields accurate count estimation on a practical level.

Analyzing a larger number of frames might lead to a more reliable estimation of cell counts, but we concluded that $15 \mathrm{fps} \times 5 \mathrm{~s}$ gives adequate sample size, as the standard deviation of the final estimation was sufficiently small (Figure $3 B$ ). The average processing time was $54 \mathrm{~s}$ per sample when a common personal computer (Intel ${ }^{\circledR}$ Core $^{\text {'" }}$ i5-5200U, RAM 8GB) was used (data not shown).

The lower limit of effective counting range was around $1 \times 10^{5}$ cells $/ \mathrm{ml}$, since the number of parasites in the field of view must be sufficiently high to give a statistically precise cell count (Supplemental

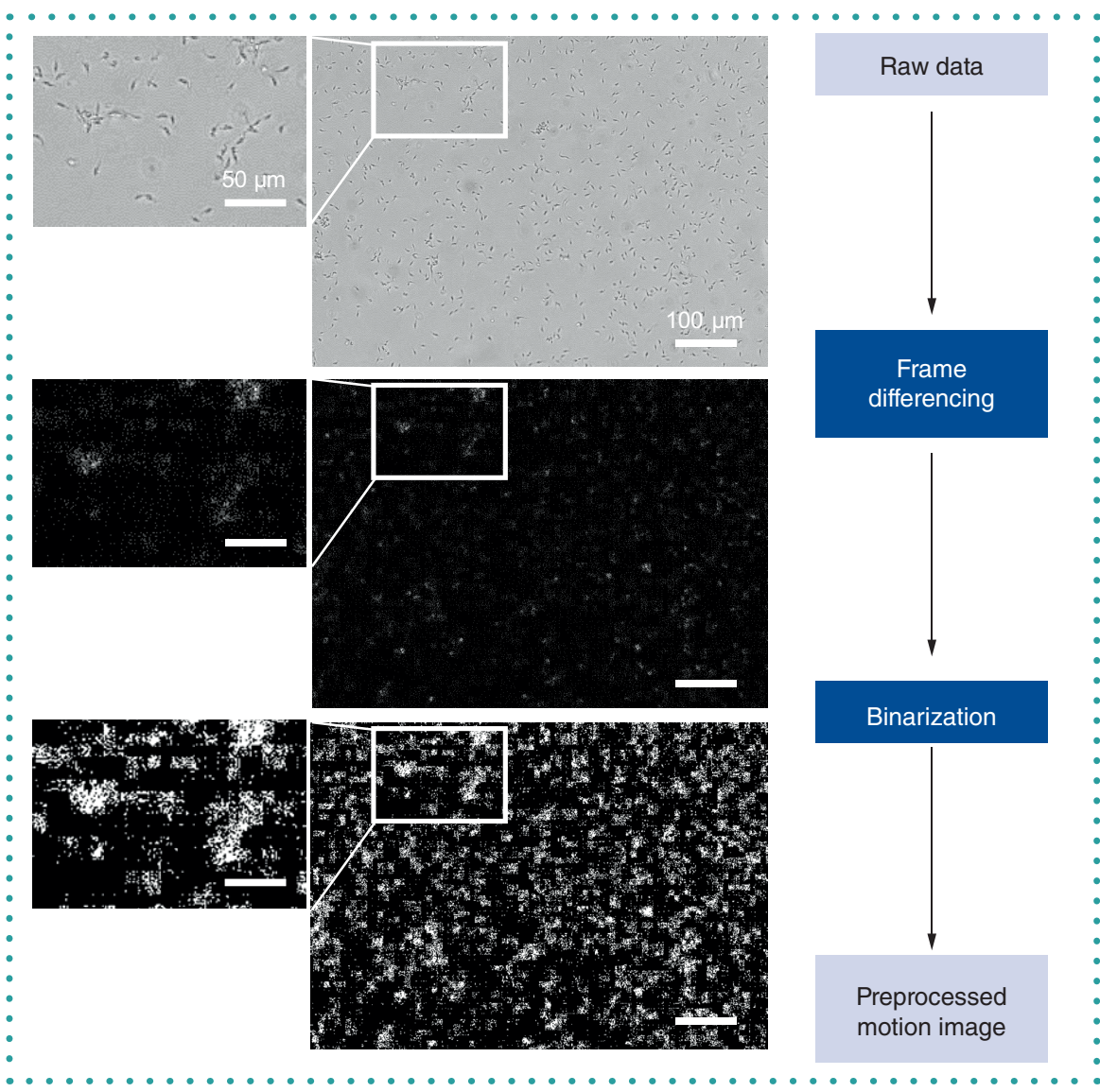

Figure 2. Flow of image preprocessing. Top panel represents original data captured as a microscopy video image. The middle panel shows the image after static background subtraction. The bottom panel is the image after binarization, which will be used in the feature extraction step.

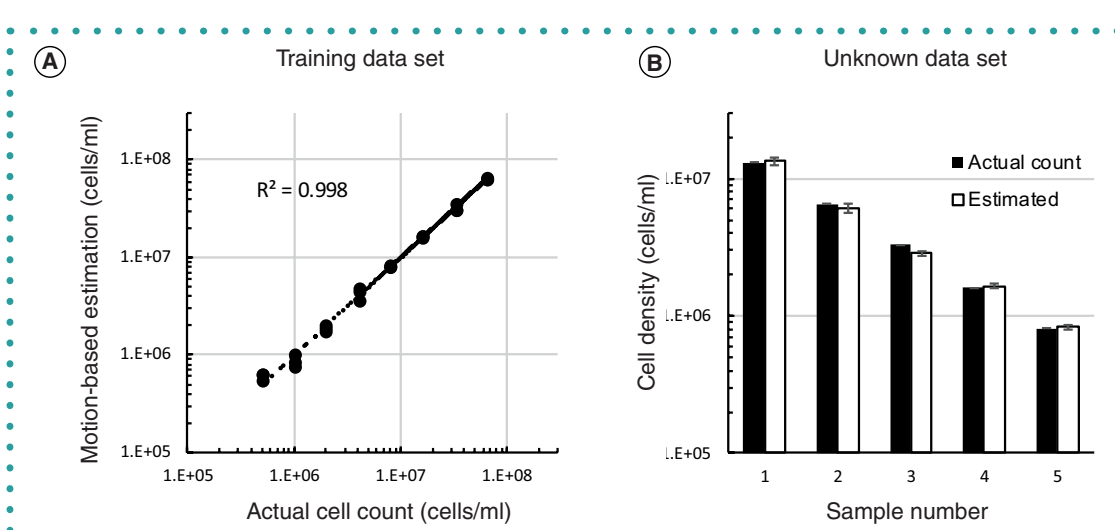

Figure 3. Validation of the motion-based cell-counting software. (A) The same motion images used as a training dataset were analyzed as a testing dataset. Three locations were selected in each well to account for the variability in cell dispersion. Coefficient of determination $\left(R^{2}\right)$ between actual cell counts and machine-estimated cell counts is shown. (B) 'Unknown' video images were analyzed as a testing dataset, based on the statistical model obtained from the training in (A).

Table 1). The upper limit of the effective counting range was around $1 \times 10^{8}$, as parasites cover the entire bottom surface of the well and saturate the view above that cell density (Figure $3 A$ and data not shown). This effective range conveniently covers the log phase of the parasite's growth curve [8], where most microbiological experiments would be conducted. In addition, the upper limit of $1 \times 10^{8}$ cells $/ \mathrm{ml}$ is considerably higher, compared to the $1 \times 10^{7}$ limit of most commercially available cell counters. This 
Figure 4. Counting rosette-rich samples.

(A) Images of $T$. cruzi epimastigote cultures containing (left) or not containing (right) cell aggregates. Arrows indicate rosettes. (B) The same motion images used as a training dataset for rosette-rich samples were analyzed as a testing dataset. Three locations were selected in each well to account for the variability in cell dispersion. Coefficient of determination $\left(R^{2}\right)$ between actual cell counts and machineestimated cell counts is shown. (C) 'Unknown' rosette-rich video images were analyzed as a testing dataset, based on the statistical model obtained from the training in (B).
Figure 5. Quantitation of fluorescent parasites. (A) Bright field (upper) and fluorescence (lower) microscopy images of the mixture of wildtype and mDsRed-expressing T. cruzi epimastigotes. Ratio of fluorescent and wildtype parasites are indicated at the bottom. (B) Machine-calculated cell counts of parasites in (A). The number of fluorescent parasites in 0:4 was assumed as zero without machine estimation, because it is out of effective counting range.

- higher effective range allows the usage of undiluted high-density cultures for video capturing. Since our method does not require any specific counting slides or application chambers, it eliminates the need to make an aliquot from the original culture. These features are advantageous, especially in a high-throughput phenotypic screening in drug discovery research.

\section{Application to aggregate-rich samples}

Epimastigotes of $T$. cruzi tend to develop cell aggregates of various sizes and shapes, called rosette (Figure 4A). The presence of rosette is a challenge for standard still image-based cell counting systems, as those aggregates are too dense for the commonly available cell image declustering software to resolve (Supplemental Figure 1).

(A)
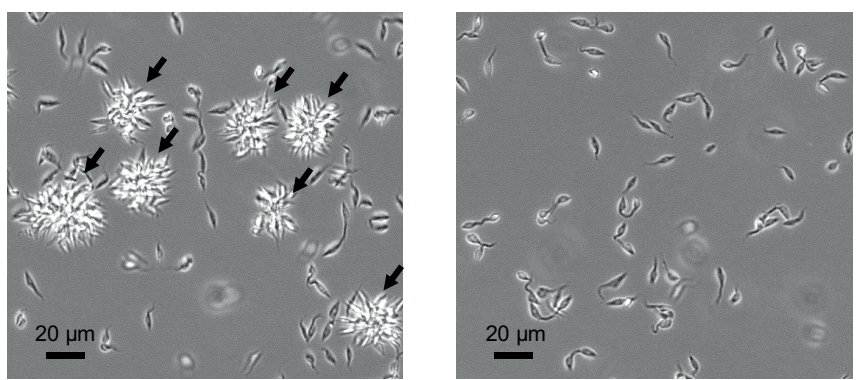

(B)

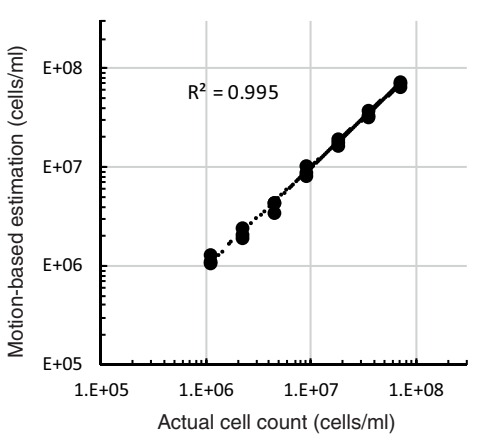

(C)

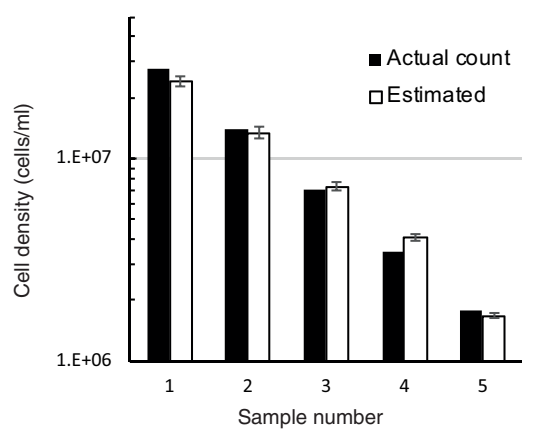

(A)

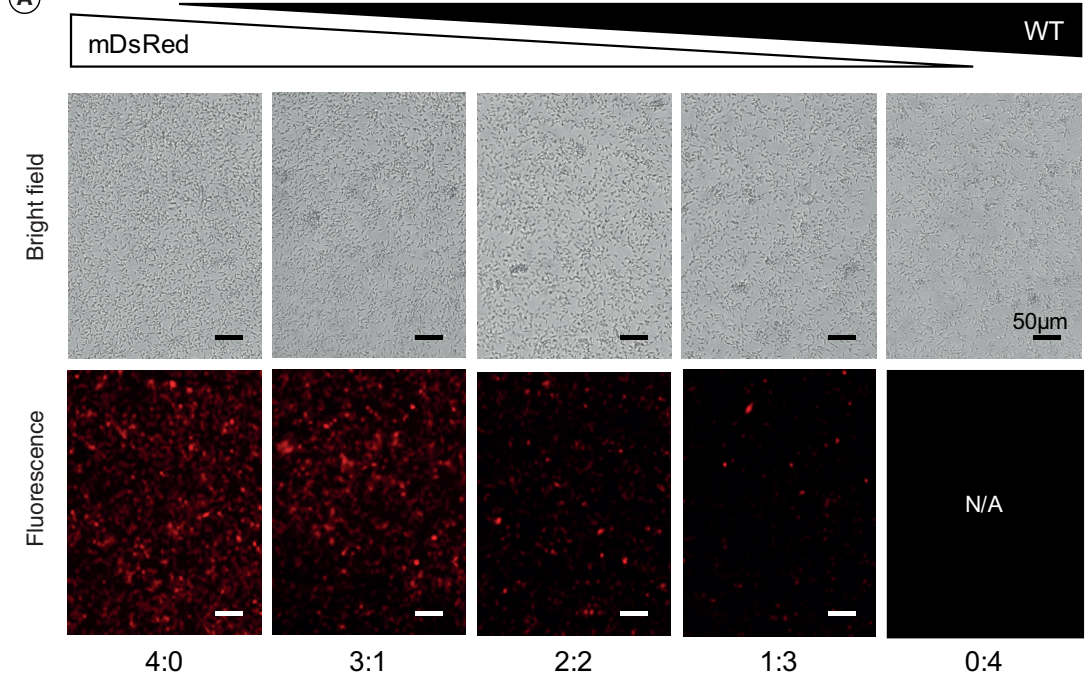

(B)

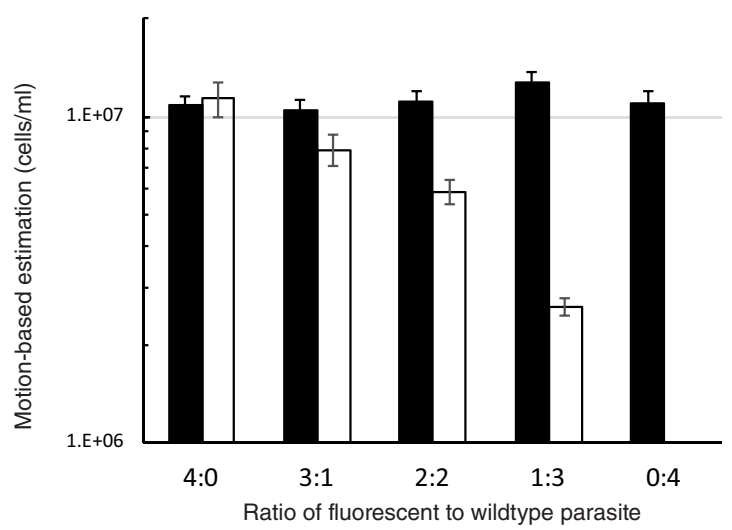

-Bright field

口Fluorescence 
Our count estimation approach is to correlate the visual noisiness of the parasite culture to the cell density, instead of counting the physical number of individual parasites itself. When the same rosette-rich samples used for the machine learning were analyzed as testing data, actual cell count and motion-based estimation showed good correlation $\left(R^{2}=0.995\right)$ (Figure 4B). Estimated count of unknown rosette-rich samples was also in good agreement with the hand-counted cell density $\left(R^{2}=0.990\right)$ (Figure 4C), suggesting that motion image recognition based on the CHLAC feature is useful in analyzing aggregate-prone parasites as well. This method provides a practical alternative as a quick estimation of the cell density of otherwise troublesome cell cultures. Eliminating the need to break apart parasite cell clumps prior to the counting gives additional advantage to our method over conventional counting from a safety perspective, since it reduces the chance of accidenta contact with a pathogenic parasite while preparing the sample for counting.

\section{Application to fluorescent parasites}

We next tested to see whether the counting program can be applied to video images taken under a fluorescence microscope. Quantitation of the fraction of fluorescent parasites in the mixture of nonfluorescent parasites can potentially be useful in monitoring transfection efficiency or knockout efficiency by using a fluorescent protein marker.

Training images were made from dilution series of $\mathrm{mDs}$ Red-expressing parasites of known cell density. For the testing dataset, wildtype and mDsRed fluorescent parasites were mixed at the ratio of $4: 0,3: 1,2: 2,1: 3$ and $0: 4$, in a total of $1 \times 10^{7}$ cells $/ \mathrm{ml}$ (Figure 5A). Motion-based cell counts were calculated separately for bright field images and for fluorescent images, based on the statistical models generated by their respective study sets. Machine-estimated cell counts in fluorescent images for 4:0, 3:1, 2:2 and 1:3 samples were $1.1 \times 10^{7}, 7.9 \times 10^{7}, 5.9 \times 10^{7}$, and $2.6 \times 10^{7}$, respectively, which are not too far from the actual cell density (Figure 5B, white bars). As expected, motion-based cell counts in bright field images yield a relatively constant number
(A)

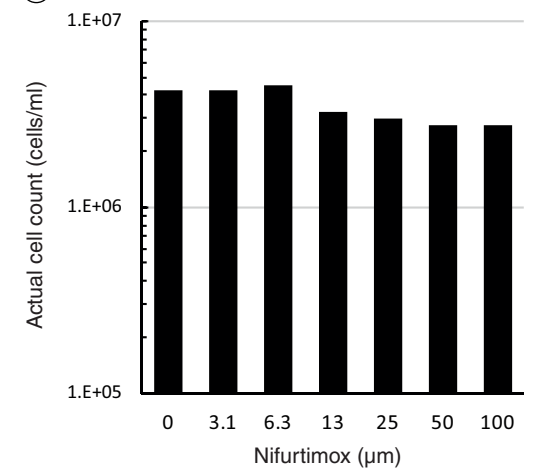

(B)

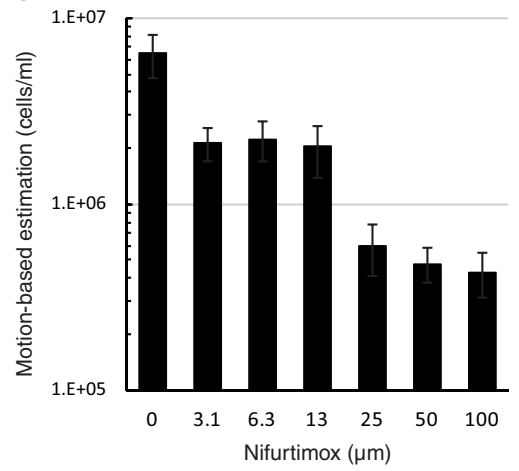

(C)

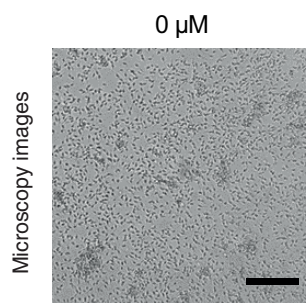

$3.1 \mu \mathrm{M}$
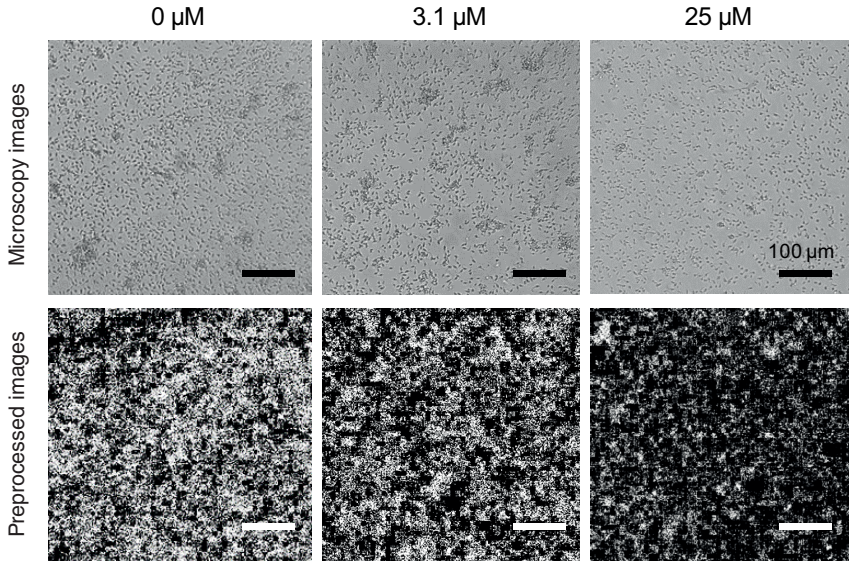

Figure 6. Reduced motility in drug-treated parasites reflected in motion-equivalent cell counts. (A) Hand-counted actual cell density of parasites incubated with indicated concentration of nifurtimox for 2 days. (B) Machine-estimated cell counts of the same parasite cultures as (A). (C) Microscopy (upper) and preprocessed (lower) video images of nifurtimox-treated T. cruzi culture. Still images from $0,3.1$ and $25 \mu \mathrm{M}$ samples are shown as representatives.

of around $1 \times 10^{7}$ cells $/ \mathrm{ml}$ across all five samples (Figure 5B, black bars). The percentages of fluorescent parasites in each sample were $104,76,53$ and $21 \%$ by machine estimation, which are reasonably close to the theoretical values of 100,75 , 50 and $25 \%$, respectively. These results suggest that video images taken under a fluorescence microscope can also be effectively used as the input data.

\section{Loss of motility as a reflection of drug efficacy}

So far, all the above case studies assume that parasites are healthy and actively wiggle their flagella. However, in the case of drug screening or knockout assays, dying parasites may become less active or even become motionless while still surviving. We tested whether this behavioral change can be captured as a difference in apparent cell density in our motion-based counting system.

Nifurtimox is a chemotherapeutic agent against Chagas' disease [9]. We added an increasing amount of nifurtimox $(3.1,6.3,13,25,50$ and $100 \mu \mathrm{M}$ final) to the parasite culture, and recorded the video images after 2 days of incubation. In terms of the actual cell count, there was only a slight decrease above $13 \mu \mathrm{M}$ nifurtimox, compared with the $0 \mu \mathrm{M}$ control (Figure 6A). Meanwhile, we observed that the parasites became torpid even with small amount of nifurtimox, which is apparent in the preprocessed motion images that reflect activeness of the cells (Figure 6C). Accordingly, in machine-estimated cell counts, a noticeable decrease started from as low as 3.1 $\mu \mathrm{M}$ nifurtimox, and significant reduction was observed 
(A)

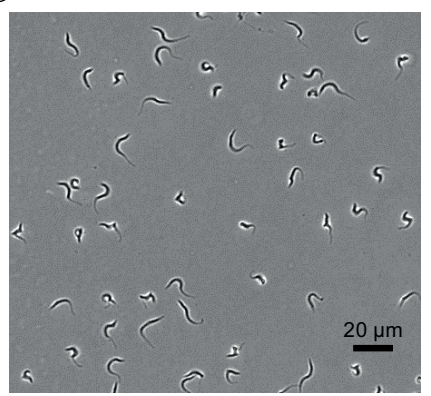

(C)

Training data set

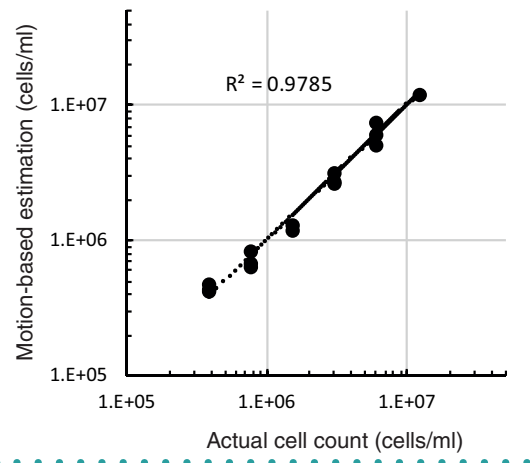

(B)

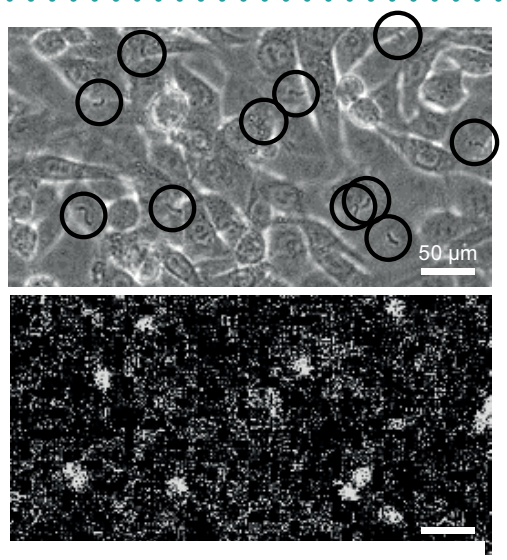

(D)

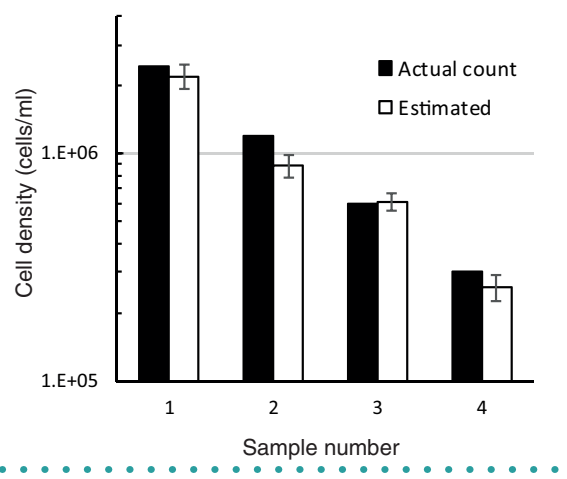

Figure 7. Counting trypomastigotes of T. cruzi. (A) Image of T. cruzi trypomastigotes under bright field without host mammalian cells. (B) Bright field image (upper) and preprocessed motion image (lower) of trypomastigote-host co-culture. Circles indicate the position of visually confirmed trypomastigotes. (C) The same motion images used as a training dataset were analyzed as a testing dataset. Three locations were selected in each well to account for the variability in cell dispersion. Coefficient of determination $\left(R^{2}\right)$ between actual cell counts and machine-estimated cell counts is shown. (D) 'Unknown' video images were analyzed as a testing dataset, based on the statistical model obtained from the training in (C).

D above $25 \mu \mathrm{M}$ (Figure 6B). This result shows that the motion-equivalent cellcounting method reflects drug efficacy more sensitively than the actual cell count.

It must be noted that this methodology has limitations in estimating the number of active parasites in the mixture of dead cells. If there are live parasites present, the dead ones do not stay completely motionless due to physical contact and stirring of the surrounding medium caused by the active parasites (data not shown). Nonetheless, the motion-based method potentially allows us to extract useful information from drug screening assays or from knockout assays, as some compounds or knockout phenotypes may not kill the parasite immediately, but impair their motility [10], in which case the effect on phenotype cannot be quantitated by conventional cell counting methodologies.

\section{Quantitation of the infectious form of \\ T. cruzi in a host co-culture}

T. cruzi has distinct developmental stages with significant morphological and behavioral differences [3]. Trypomastigote is the infectious form of $T$. cruzi, which is highly motile and has a slender, flexible body (Figure 7A). Unlike epimastigotes, trypomastigotes do not form rosettes, so it is relatively simple to count this stage of parasite by itself. However, trypomastigotes are normally maintained in a co-culture with host mammalian cells, so the challenge of counting this stage of parasite is to identify trypomastigotes surrounded by the visual noise of the host cell culture.
To this end, we took advantage of the nature of the motion-based counting system, which can virtually ignore the static objects as background (Figure 7B). To prepare the sample motion images, known cell densities of trypomastigotes were applied onto host 3 T 3 cells in 96 wells, and video images were captured in the same manner as previous experiments. When the same video images used for the training dataset were analyzed as the testing data, actual cell counts and machineestimated cell counts showed good correlation $\left(R^{2}=0.979\right)$ (Figure $\left.7 C\right)$. Estimated cell counts of unknown samples were also in good agreement with hand-counted cell densities (Figure 7D). These results suggest that the motion-based counting method is useful in identifying and quantitating motile parasites in the presence of co-cultured host cells without additional sample or data pretreatment steps.

\section{FUTURE PERSPECTIVE}

We demonstrated that the motion pattern recognition method based on the CHLAC feature can be applied to the cell-counting system of $T$. cruzi. The fact that our software successfully estimated cell densities of two different stages of $T$. cruzi with distinct appearances and behavioral characteristics from each other makes it plausible that this approach may be applicable to cell-count estimation of a wide range of free-swimming microorganisms. By eliminating the need for a specific counting chamber and by tolerating highly dense samples, our method minimizes the handling steps required for sample preparation. We could potentially reduce the hands-on time even further by combining with an automated recording system to capture microscopy video images in 96-well format, dramatically improving the throughput. These possibilities not only reduce the burden of labor, but also provide a safer experimental option for studying pathogenic parasites. Altogether, our present study suggests that the motionbased counting system has a potential for becoming a beneficial tool to safely and efficiently conduct phenotypic assays of clinically relevant microbes.

\section{AUTHOR CONTRIBUTIONS}

The software was developed by $\mathrm{HN}$ and $\mathrm{HS}$. $\mathrm{KF}$ contributed to the conception of the study. 
YT conducted experiments and wrote the manuscript. MD contributed to data interpretation. All authors worked on correction of the manuscript and approved the final submission.

\section{ACKNOWLEDGMENTS}

We thank Dr Kenji Hirayama and Dr Tetsuo Yanagi for their input in the early phase of this project, Atsuko Furukawa for technical assistance, and Dr Yukie Akutsu for the helpful discussion.

\section{FINANCIAL \& COMPETING INTERESTS DISCLOSURE}

This work was supported in part by KAKENHI Grant number: 18K15141. The authors have no other relevant affiliations or financial involvement with any organization or entity with a financial interest in or financial conflict with the subject matter or materials discussed in the manuscript apart from those disclosed.

No writing assistance was utilized in the production of this manuscript.

\section{OPEN ACCESS}

This work is licensed under the AttributionNonCommercial-NoDerivatives 4.0 Unported License. To view a copy of this license, visit http://creativecommons.org/licenses/ by-nc-nd/4.0

\section{SUPPLEMENTARY DATA}

To view the supplementary data that accompany this paper please visit the journal website at: www.future-science. com/doi/suppl/10.2144/btn-2018-0163

\section{REFERENCES}

1. World Health Organization (WHO). Fact sheet: Chagas disease (American trypanosomiasis). Washington, DC USA, (updated 2017 Mar; cited 2017 Aug) (2017).

2. Apt W. Current and developing therapeutic agents in the treatment of Chagas disease. Drug Des. Devel. Ther. 4, 243-253 (2010).

3. Clayton J. Chagas disease 101. Nature. 465(n7301_ supp), S4-S5 (2010).

4. Vazquez MP, Levin MJ. Functional analysis of the intergenic regions of TcP2beta gene loci allowed the construction of an improved Trypanosoma cruzi expres sion vector. Gene 239(2), 217-225 (1999).

5. Shaw AK, Kalem MC, Zimmer SL. Mitochondrial gene expression is responsive to starvation stress zi. mSphere 1(2), doi:10.1128/mSphere.00051-16 (2016).
6. Kobayashi T, Hosaka T, Mimura S, Hayashi T, Otsu N HLAC Approach to automatic object counting. In: ECSIS Symposium on Bio-inspired, Learning, and Intelligent Systems for Security (BLISS). 40-45 (2008).

7. Kobayashi T, Otsu N. Action and simultaneous multiple-person identification using cubic higher-order loca auto-correlation. In: International Conference on Pattern Recognition (ICPR). 4, 741-744 (2004).

8. De Paula Lima CV, Batista M, Kugeratski FG et al. LM14 defined medium enables continuous growth of Trypanosoma cruzi. BMC Microbiol. 14(1), 238 (2014).

9. Rassi A Jr, Rassi A, Marin-Neto JA. Chagas disease. Lan cet 375(9723), 1388-1402 (2010).

10. Lander N, Li Z-HH, Niyogi S, Docampo R. CRISPR/ Cas9-induced disruption of paraflagellar rod protein 1 and 2 genes in Trypanosoma cruzi reveals their role in flagellar attachment. MBio. 6(4), e01012 (2015). 